\title{
Targeting of IL-2 and GM-CSF immunocytokines to a tumor vaccine leads to increased anti-tumor activity
}

\author{
PHILIPPE FOURNIER ${ }^{1}$, MAXIMILIAN AIGNER $^{1}$ and VOLKER SCHIRRMACHER ${ }^{1,2}$ \\ ${ }^{1}$ Division of Cellular Immunology, German Cancer Research Center, Heidelberg; ${ }^{2}$ IOZK, Cologne, Germany
}

Received December 21, 2010; Accepted February 2, 2011

DOI: 10.3892/ijo.2011.976

\begin{abstract}
Fusion proteins combining antibodies with cytokines such as IL-2 and GM-CSF appear to be promising reagents for tumor therapy. In this study, we combined such immunocytokines with the tumor vaccine ATV-NDV consisting of irradiated tumor cells infected with Newcastle disease virus (NDV). The two fusion proteins bsF-GMCSF and tsHN-IL2-GM-CSF, binding, respectively, to the viral fusion protein $(F)$ or to hemagglutinin-neuraminidase $(\mathrm{HN})$ expressed on the surface of the vaccine cells and containing GM-CSF or GM-CSF and IL-2-activities were produced by recombinant antibody technology. The purified molecules showed the expected binding specificity and biological activity inherent to the respective cytokine. Using a newly established in vitro tumor neutralisation assay (TNA), we showed improved antitumoral effect through tumor growth inhibition when human peripheral blood mononuclear cells from healthy donors were stimulated with immunocytokine modified versus non-modified tumor vaccine cells. These effects induced by the fusion proteins, in the presence of a suboptimal $\mathrm{T}$ cell activation signal 1 provided by bsHN-CD3, occured only when these were bound to the tumor vaccine. Furthermore, it was shown that $\mathrm{CD} 14^{+}$monocytes could be activated by the GM-CSF cytokine fused within the recombinant proteins and that they contributed essentially to the antitumor effect in the TNA. The data presented here suggest an easy way for a broad clinical development and application of tumor-targeted IL-2- and GM-CSF-based immunocytokines based on the associated increase of anti-tumor activity mediated by $\mathrm{T}$ cells and monocytes.
\end{abstract}

\section{Introduction}

Most tumors are only very weakly immunogenic. That means that it is difficult for the immune system to recognize tumor

Correspondence to: Professor Volker Schirrmacher or Dr Philippe Fournier, German Cancer Research Center (DKFZ), Im Neuenheimer Feld 280, 69120 Heidelberg, Germany

E-mail: v.schirrmacher@dkfz.de

E-mail: p.fournier1@yahoo.de

Key words: fusion protein, antitumor vaccination, costimulation, interleukin-2, granulocyte macrophage colony stimulating factor, cytokines, bystander effect cells as foreign and to mount a response leading to rejection of a tumor. In addition, tumors can develop a variety of strategies to evade immune surveillance. Active-specific immunotherapy (ASI) makes use of tumor vaccines which contain tumor antigens and adjuvants. Following this strategy, we have developed in the past 10 years the human autologous tumor vaccine modified by infection with Newcastle disease virus which is called ATV-NDV.

For virus infection of tumor cells, we used an avirulent and non-lytic NDV strain (Ulster) so that the tumor cells remain viable for 24-48 h. For the production of the ATV-NDV vaccine, the tumor cells are $\gamma$-irradiated with 200 Gy to prevent cell division (1), and infected with a low dose of NDV Ulster [32 hemagglutination units (HU) $/ 10^{7}$ tumor cells] for $1 \mathrm{~h}$ at $37^{\circ} \mathrm{C}$. This vaccine is then applied intra-dermally (i.d.) to cancer patients $(2,3)$. Phase II clinical trials which were performed in patients having different tumor entities such as breast cancer, colon cancer, melanoma or glioblastoma showed the efficacy of the vaccine. The post-operative treatment led to an increase in the percentage (20-36\%) of long-term (2-5 years) survivors when compared to controls, summarized in ref. (2). Recently, results of a randomized clinical study in colon cancer patients have corroborated these findings. They demonstrate a $30 \%$ increase of survivors even after a period of 10 years (4).

The rationale of the ATV-NDV vaccine is to combine autologous TAAs with danger signals $(5,6)$ and costimulatory signals $(7,8)$. The present study aimed at modifying this tumor vaccine further in order to activate anti-tumor activity also from naive $\mathrm{T}$ cells (tumor specific or not). A possiblity to further improve this vaccine appears via the supplementary administration of immunomodulatory cytokines, such as interleukin-2 (IL-2) and the granulocyte macrophage colony stimulating factor (GM-CSF) to support the immune system to fight the tumor. To this end, we constructed various NDV-specific fusion proteins that could easily be attached to the ATV-NDV vaccine.

In the present study, we target immunocytokines not to tumor-associated antigens (TAAs) but to viral antigens [hemagglutinin-neuraminidase $(\mathrm{HN})$ and fusion protein $(\mathrm{F})]$ which are expressed on tumor cells (9) and can thus serve as universal targets. Both viral molecules $\mathrm{HN}$ and $\mathrm{F}$ become selectively over-expressed on tumor cells infected by NDV (10). The advantages of this concept are i) that these 2 viral proteins can serve as universal anchor molecules for immu- 
nocytokines and ii) that viruses can activate additional innate anti-tumor immune mechanisms. We describe here HN- and F-specific fusion proteins. They can be used to modify all types of human cancer that are susceptible to infection by NDV.

One of the cytokines we targeted to tumor cells is IL-2. It is a $15-20-\mathrm{kDa}$ glycoprotein which is secreted by activated helper $\mathrm{T}$ cells $(11,12)$ and has pleiotropic functions. It promotes T-cell proliferation and activates both $\mathrm{T}$ cells as well as NK cells to cytotoxic effector cells. IL-2 was used for the generation of lymphokine-activated killer (LAK) cells from peripheral blood lymphocytes or for the expansion of tumor infiltrating lymphocytes (TIL). Intravenous application of high doses of IL-2 in melanoma and renal carcinoma patients led to $8 \%$ of the patients to a complete remission and $9 \%$ to a partial tumor regression (13). Such treatments, however, are associated with high systemic toxicity. Side-effects include the capillary leak syndrome (CLS) $(14,15)$, hypertension, weight gain, respiratory failure and neurotoxicity (16). To avoid these unwanted side-effects, the dose of IL-2 needs to be reduced. Tumor targeting of IL-2 appears as a very interesting strategy to avoid systemic IL-2 application (17).

The second cytokine we targeted to tumor cells is GM-CSF. It is a $20-25 \mathrm{kDa}$ glycoprotein which is produced by $\mathrm{T}$ cells, macrophages, fibroblasts and IL-1-stimulated endothelial cells (12). GM-CSF promotes the recruitment and differentiation of granulocytes, macrophages and eosinophil progenitor cells (18). In addition, GM-CSF has a positive influence on dendritic cells, since it improves their phagocytic activity $(19,20)$ and upregulates expression of antigen-presenting molecules such as MHC (classes I and II) (21) and of costimulatory molecules (22). These effects result in an improvement of the T-cell stimulation. Systemic administration of high doses of GM-CSF can lead to leukocytosis, swelling of the liver or spleen and may lead to hemorrhagy of the lung as shown in rats (23). To reduce side-effects without reducing the antitumor effect, the cytokines IL-2 and GM-CSF have been administered, in recent years, locally to the tumor following two strategies. One approach was to infect tumor cells ex vivo with recombinant viruses that produce these cytokines (e.g. replication-retroviruses or adenoviruses) in situ when the vaccine is applied to the patient. The other approach involved the use of immunocytokines. For this, genes are fused to an antibody or an antibody fragment gene that binds to a tumortarget.

In this study, we designed and produced immunocytokines that bind to NDV (HN or F) at the surface of the NDV-infected tumor vaccine. We show that IL-2 and GM-CSF-containing fusion proteins can be produced in sufficient quantity and that these exhibit the correct binding specificity and functional activity. Modification of a NDV-infected tumor vaccine by attachment of such immunocytokines led to a stronger direct stimulatory activity of human PBMC and to the generation of a strong bystander anti-tumor activity in vitro. Such activities were not observed with the immunocytokines in the absence of the tumor vaccine. It also required the presence of a certain level of $\mathrm{T}$ cell activation which was provided via an anti-CD3 bispecific antibody molecule (bsHN-CD3). By combining ATV-NDV with NDV-specific immunocytokines, we can obtain a new second generation tumor vaccine exposing IL-2 and/or GM-CSF alone or in combination with anti-CD3 (as signal 1).

We also demonstrate that monocytes play an important role in the bystander antitumor activity mediated through the tumor-targeted GM-CSF. Moreover, we show that GM-CSF and IL-2 can be targeted together in one fusion protein to tumor cells. This then leads to a stronger bystander antitumor activity than exerted via GM-CSF alone.

\section{Materials and methods}

Cell lines. All cell lines were obtained from the tumor cell bank of the German Cancer Research Center (DKFZ, Heidelberg, Germany). Cell culture media were supplemented with $2 \mathrm{mM}$ L-glutamine, $10 \mathrm{mM}$ HEPES, $100 \mathrm{U} / \mathrm{ml}$ penicillin and $100 \mu \mathrm{g} / \mathrm{ml}$ streptomycin (all purchased from Gibco Invitrogen, Karlsruhe, Germany, unless otherwise mentioned). The human breast carcinoma cell line MCF-7 was cultured in DMEM with $10 \%$ fetal calf serum (FCS). The murine fibroblast line Ltk, its HN cDNA (Ltk-HN.A2, here called Ltk-HN) (7), its F cDNA (Ltk-F.B9, here called Ltk-F) and its HN+F cDNAs Ltk- $(\mathrm{HN}+\mathrm{F})$ transfectants were cultured in RPMI-1640 medium with $10 \%$ FCS. The human T cell lymphoma line CTLL was grown in ISCOVES medium containing $10 \%$ FCS and $25 \mathrm{U} / \mathrm{ml}$ recombinant human IL-2 (Chiron Therapeutic, Emeryville, USA). The human bone marrow erythroleukemia cell line TF-1 and the murine cytotoxic T-cell clone CTLL were grown in RPMI medium with 5\% FCS and implemented with $2.5 \mathrm{ng} / \mathrm{ml}$ of human recombinant GM-CSF (Sandoz, Ismmaning, Germany). Before each experiment, the CTLL and TF1 cells were cultured overnight without any cytokine. Recombinant human IL-2 and GM-CSF proteins were used as standards for the quantification of the cytokine activity based on the proliferation of the CTLL and TF1 cells. Transfected $\mathrm{CHO}$ cells were cultured in $\alpha$-MEM containing $5 \%$ dialysed FCS (dFCS, Biochrom, Krefeld, Germany) and $10 \mathrm{nM}$ methotrexate (Calbiochem, Schwalbach, Germany). The human lymphoblastoid $\mathrm{T}$ cell line Jurkat was cultured in RPMI with $5 \%$ FCS.

Human peripheral blood mononuclear cells (PBMC) were isolated from buffy coat or from fresh heparinized blood of healthy donors via centrifugations using Ficoll-Paque (Amersham Biosciences, Freiburg, Germany) centrifugation. Monocyte isolation and depletion were performed via magnetic separation of cells labelled with mouse mAbs against human CD14, and incubated with Dynabeads coated with human IgG4 against mouse IgG Fc according to manufacturer's instructions (Miltenyi, Bergisch Gladbach, Germany).

Tumor vaccine preparation and production of recombinant fusion proteins. For preparation of the tumor vaccine, the avirulent, non-lytic strain Ulster of NDV was used. Origin, maintenance and purification of the virus have been described previously (24). Quantification was performed by hemagglutination (HA) assay. The smallest virus concentration leading to visible agglutination of sheep erythrocytes is defined as 1 hemagglutination unit (HU). MCF-7 cells were $\gamma$-irradiated with 200 or 100 Gy, respectively, via a 137Cs source (Gammacell 1000, Atomic Energy of Canada, Chalk River, Canada) and then modified for $1 \mathrm{~h}$ at $37^{\circ} \mathrm{C}$ with $100 \mathrm{HU}$ NDV per $1 \times 10^{7}$ 


\section{Recombinant fusion proteins}

bsF-GMCSF

(774)

A
tsHN-IL2-GMCSF

(766)

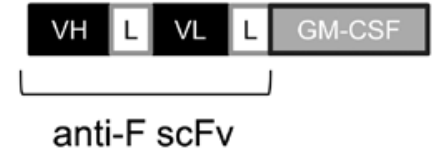

B

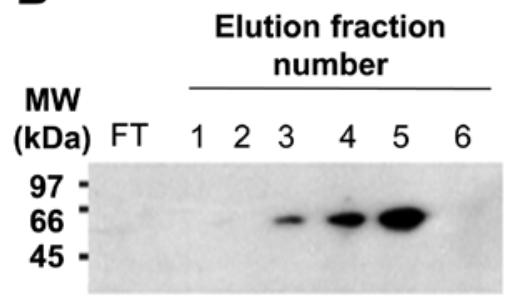

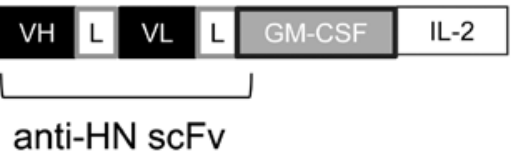

Elution fraction number

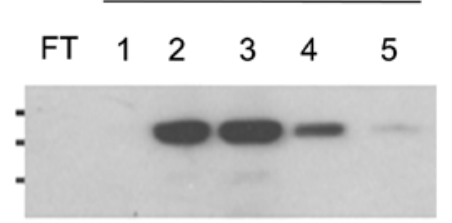

Figure 1. Biochemical characterization of the fusion proteins bsF-GMCSF (no. 774) and tsHN-IL2-GMCSF (no. 766). (A) Structure of the fusion protein bsF-GMCSF (no. 774) and tsHN-IL2-GMCSF (no. 766). (B) Western blot analysis of both proteins. For the analysis of the size of the two recombinant fusion proteins produced, $25 \mu \mathrm{l}$ of each individual fraction obtained from the purification column during elution were mixed 1:2 with a reducing SDS sample buffer and incubated for $5 \mathrm{~min}$ at $95^{\circ} \mathrm{C}$. Then $20 \mu \mathrm{l}$ of each sample were applied on two $12.5 \%$ SDS acrylamide gels. After migration, the proteins of the gel were transferred by Western blotting to a nitrocellulose membrane and stained with anti-Flag primary antibody (concentration: $10 \mu \mathrm{g} / \mathrm{ml}$ ). The development of the membrane was performed using a secondary antibody (concentration: $0.08 \mu \mathrm{g} / \mathrm{ml}$ ) coupled to peroxidase and ECL substrate. The proteins were visible on X-ray film. L, Gly4Ser; FT, flow through; MW, molecular weigth; kDa, kiloDalton.

cells (VN: MCF-7-NDV), as described (25). Uninfected vaccine cells (V) served as control.

The plasmids encoding the bispecific constructs bsHN-CD3 (no. 242) (25), as well as bsHN-IL-2 (no. 356) (26) have been described. The plasmids expressing the new constructs which are shown for the first time in this study were constructed by combining DNA coding for the scFv specific for the HN or $\mathrm{F}$ protein of NDV with cDNAs coding for the cytokine(s). The cDNA coding for the the human IL-2 protein was kindly provided by Dr M. Zöller (DKFZ, Heidelberg, Germany). The cDNA of GM-CSF was derived from the plasmid pCD-hGMCSF which was purchased from ATCC (Rockville, USA). The amplification of the DNA plasmids was performed in TOP-10 bacteria. Transfection of the plasmids into dCHO cells was carried out by electroporation (BioRad, Munich, Germany). Clones that were stably expressing the desired fusion protein were selected by limiting dilution technique. The production was conducted in high density cell culture systems (Integra Biosciences, Fernwald, Germany).

Antibodies and flow cytometry. The mAbs specific for His6-tag (mouse IgG1) and Flag-tag (mouse IgG2b) were obtained respectively from Dianova (Hamburg, Germany) and Sigma-Aldrich (Schnelldorf, Germany). Horse raddish peroxidase (HRP)-conjugated goat anti-mouse IgG was from Jackson ImmunoResearch (Cambridgeshire, England). PE-labelled goat $\left.\mathrm{F}(\mathrm{ab})_{2}\right)_{2}$ anti-mouse $\mathrm{IgG}$ was from Southern Biotech (Birmingham, USA). Anti-human CD14 mouse IgG2a, antihuman CD116 biotin (mouse IgM) and streptavidin-PE were all purchased from BD Pharmingen (Heidelberg, Germany).
Before any antibody labelling, human cells were first blocked with endobulin (human intact IgG, $50 \mu \mathrm{g} / \mu \mathrm{l}$, purchased from Baxter, Unterschleissheim, Germany). After staining, the cell suspensions were analyzed with a FACSCalibur fluorescence analyzer (Becton-Dickinson, Heidelberg, Germany). Propidium iodide (PI, $0.5 \mu \mathrm{g} / \mu \mathrm{l}$, Sigma-Aldrich, Schnelldorf, Germany) was added to all cell suspensions immediately before the FACS analysis to exclude dead cells. The data were analyzed using the FlowJo software (Tree Star, Ashland, USA).

Tumor neutralization assay (TNA) in 48-well plates. The assay system was adjusted from (27). The experimental tests were performed in triplicates in 48-well round-bottom microtiter plates. Briefly, $14 \times 10^{3}$ live MCF-7 tumor cells (in a volume of $150 \mu \mathrm{l}$ ) were added to the wells and incubated for $4 \mathrm{~h}$ in culture medium until they became adherent. Effector cells were freshly isolated PBMC (or a defined subpopulation) derived from healthy donors. The effector cells were added in a volume of $100 \mu \mathrm{l}$ to the wells at an effector to tumor cell ratio of 5:1 $\left(30 \times 10^{3}\right.$ cells/well). Then, $6 \times 10^{3}$ MCF-7-NDV vaccine cells in a volume of $30 \mu \mathrm{l}$ were added to the wells. The desired amounts of the fusion proteins to be tested were added in a total volume of $100 \mu \mathrm{l}$. The plates were incubated for 6-7 days (until the tumor monolayer in the control wells was confluent).

At the end of the assay, the supernatants of the 48-well TNAs were removed and the wells were carefully washed 5 times with $400 \mu \mathrm{l}$ RPMI-1640 medium (plus 5\% FCS) to remove non-adherent cells. After the last wash, the wells were filled with $200 \mu \mathrm{l}$ RPMI-1640 medium (plus 5\% FCS) and 
Table I. Summary of the characteristics of the 2 purified fusion proteins used in this study.

\begin{tabular}{lclrr}
\hline Protein & No. & \multicolumn{1}{c}{ Protein structure } & $\begin{array}{r}\text { IL-2 specific activity } \\
\text { (U/ } \mu \text { g protein) }\end{array}$ & $\begin{array}{r}\text { GM-CSF activity } \\
(\mathrm{ng} \text { eq./ } \mu \mathrm{g} \text { protein })\end{array}$ \\
\hline bsF-GMCSF & $774(2 \mathrm{G} 1)$ & Flag-VH-L-VL-L-GMCSF & 0 & 2.37 \\
tsHN-IL2-GMCSF & $766(\mathrm{H} 3)$ & Flag-VH-L-VL-IL2-L-GMCSF & 80,299 & 16.12 \\
bsHN-IL2 & 356 & VH-L-VL-IL2 & 51,852 & 0.00
\end{tabular}

This table provides a listing with some characteristics of the 2 different fusion proteins which have been used in this study. The protein number refers to the plasmid which codes for the particular protein, the clone number to the well of the 96-well plate from where the clone was isolated. The N- and C-terminal Tag sequences, such as flag tag or His6 were used for the purification of the recombinant proteins as well as to its detection in Western blot or FACS. Each fusion protein with internal reference number is characterized by its protein concentration (determined by Bradford), its HN- or F-activity, and its IL-2 or GM-CSF activity (assessed by using the IL-2-dependent cell line CTLL). The HN- or F-activity was quantified by titrating the proteins on HN- or F-expressing Ltk mouse fibroblasts followed by FACS analysis. An arbitrary activity of $10,000 \mathrm{U} / \mathrm{ml}$ was attributed to a protein dilution which stained $50 \%$ of the respective Ltk transfectants. The amplification of the DNA plasmids was performed in TOP-10 bacteria. Transfection of the plasmids into dCHO cells was carried out by electroporation (BioRad). Clones that were stably expressing the desired fusion protein were selected by limiting dilution technique. The production was conducted in high density cell culture systems (Integra Biosciences). The fusion proteins containing a His6-tag at their C-terminus were purified by immobilized metal affinity chromatography (IMAC), as described (25). The purity of the different fractions was assessed by Coomassie staining after separation of the purified proteins on $12.5 \%$ SDS-PAGE gels. The size of the molecules was checked by Western blot. The fractions exhibiting the highest purity and protein amount were pooled, dialyzed against PBS, filtered $(0.22 \mu \mathrm{m})$ and stored at $-80^{\circ} \mathrm{C}$. The protein concentration was determined with the CB-Protein Assay ${ }^{\mathrm{TM}}$ reagent (Calbiochem) and using bovine serum albumin (BSA) as reference protein. To construct the new plasmids (inducing the production of both proteins nos. 770 and 766), the scFvs specific for the HN or F protein of NDV was combined with the genes coding for the IL-2 and GM-CSF cytokines within the pERdhfr vector. The transcription of the fusion proteins was carried out on the strong CMV (cytomegalovirus) promoter. RBS (ribosome-binding sites) sequences were used to translation initiation of proteins as well as the selection markers dhfr (dihydrofolate reductase). For propagation and selection of plasmids in bacteria, this plasmid possesses one Ampicillin resistance (AMPR) and a starting point for prokaryotic replication (ori). Functional activities of the fusion proteins nos. 356,770 and 774 were determined in CTLL and TF1 assays. To this end, the proteins were serially diluted using a dilution factor of 4 . To each well was added $1 \times 10^{4}$ IL-2-dependent CTLL or $1 \times 10^{4}$ GM-CSF-dependent TF-1 cells. After an incubation of 20 (CTLL) or 48 (TF1) h, $1 \mu \mathrm{Ci}$ of ${ }^{3} \mathrm{H}$-thymidine was added into each well and incubated for a further $4 \mathrm{~h}$. The incorporated radioactivity was measured in a liquid scintillation counter. All values were measured as duplicates. Internal IL-2 or GM-CSF standards with defined concentrations in the respective cytokines allow the exact quantification of the activities of these fusion proteins. The bsHN-CD3 and bsHN-IL2 proteins were used respectively as negative and positive controls. L, Gly4Ser.

$40 \mu 1$ MTS solution (MTS Cell Titer $96^{\circledR}$ AQueous, Promega, Mannheim, Germany) was added. The plates were then incubated for $45 \mathrm{~min}$ at $37^{\circ} \mathrm{C}$ in the dark. When, in the control tumor monolayer wells (without MLTC), the colour switched from yellow to brown, the reaction was stopped by adding $40 \mu 1$ of a $10 \%$ SDS solution per well. The plates were then evaluated after transfer of $200 \mu 1$ in a 96 -well plate by reading the OD at $490 \mathrm{~nm}$ using an ELISA reader. The background was taken from 3 control wells containing only medium, MTS reagent and SDS solution. The percent tumor growth inhibition was calculated as described (27).

Statistical analysis. Differences between two means were calculated in Excel software with the Student's t-test. They were regarded as significant at $\mathrm{p}<0.05$.

\section{Results}

Production and biochemical characterisation of fusion proteins binding to NDV surface proteins and containing $I L-2$ and/or GM-CSF cytokines. The cloning strategy consisted of the insertion within the pERdhfr mammalian expression vector of a gene coding for a single chain $\mathrm{Fv}$ antibody $(\mathrm{scFv})$ directed against a surface protein of NDV. This was then linked via a sequence coding for a flexible spacer of 15 amino acids to another gene coding for GM-CSF. We also contructed a trispecific (ts) fusion protein containing GM-CSF together with IL-2 (Fig. 1A). The plasmids were then transfected by electroporation into dhfr deficient $\mathrm{CHO}$ cells and stable clones producing the expected protein were established by subcloning. The production of the recombinant proteins was performed in a high density cell culture system. For purification, the supernatants were first dialyzed and then passed through a nickel-NTA-Sepharose column. The recombinant proteins 774 and 766 (Table I) bound to the column via the His6 tag which had been introduced at the C-terminal end. After one or several washing steps, the proteins were eluted from the column by increasing the concentration of imidazole. These fractions were examined by SDS-PAGE and subsequent Coomassie staining (data not shown) and Western blot (Fig. 1B) for the determination of the quantity and purity of the proteins. The fractions with the highest and purest protein content were mixed, dialyzed against PBS, sterile filtered, aliquoted and then stored at $-80^{\circ} \mathrm{C}$.

Binding and functional properties of the fusion protein containing NDV-specific scFv and IL-2 and/or GM-CSF cytokines. The fusion proteins were further characterized for their binding properties by flow cytometry. To this end, we used several established cell lines including the mouse fibro- 


\section{Recombinant fusion proteins}

bsF-GMCSF

(774)

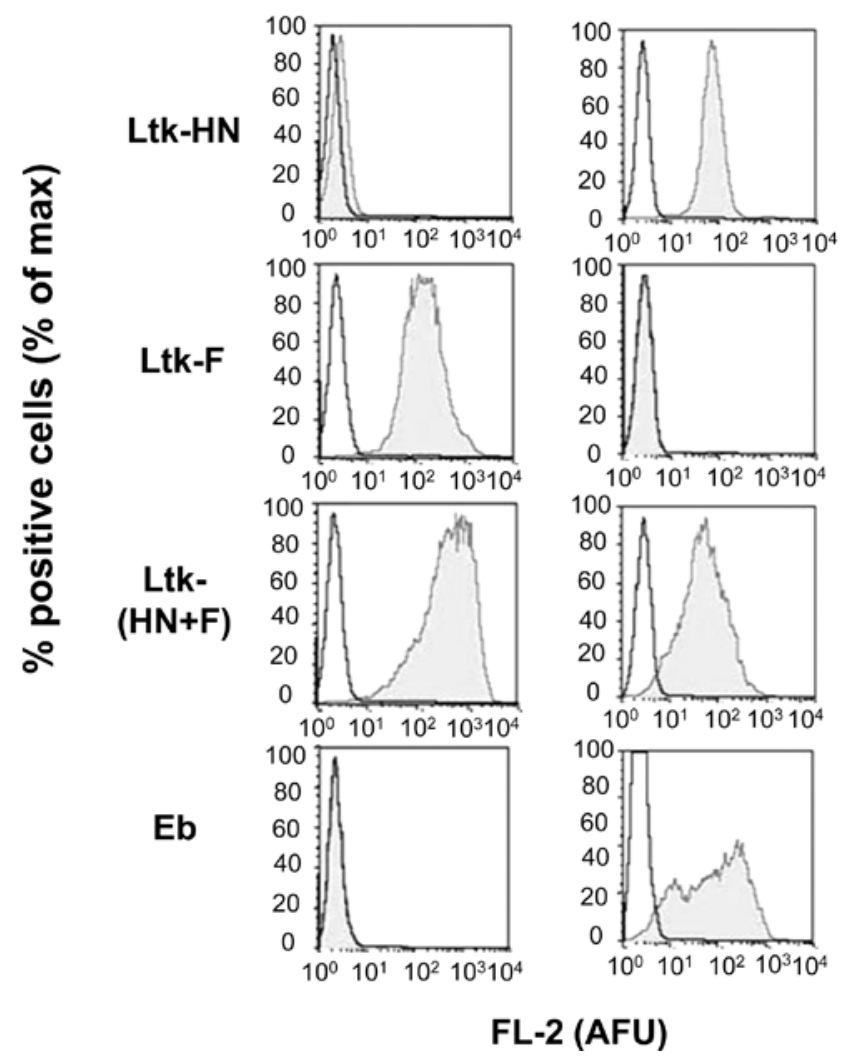

Figure 2. Binding of the fusion proteins by flow cytometry to NDV proteins and to IL-2R expressed at the cell surface. Ltk-HN, Ltk-F, Ltk-(HN+F) or Eb cells $\left(1 \times 10^{5}\right)$ were incubated for 45 min with $1 \mu \mathrm{g}$ of the protein 774 or 766. The staining was carried out with anti-His or anti-E-tag primary antibody and then with PE-conjugated goat anti-mouse secondary antibody. As a negative control, samples were only incubated with an isotype contro antibody and secondary antibody. Dead cells were excluded by PI staining.

blast Ltk cell line that was stably transfected with a plasmid coding either for the viral antigen $\mathrm{HN}$ or $\mathrm{F}$ (respectively Ltk-HN and Ltk-F) or for both proteins, Ltk-(HN+F). The results are shown in Fig. 2. Protein no. 774 bound to Ltk-F but not to Ltk-HN whereas protein no. 766 showed binding to Ltk-HN but not to Ltk-F. Both proteins bound to Ltk-(HN+F) (Fig. 2) and also to MCF-7 human carcinoma cells which had been irradiated (200 Gy) and modified through $1 \mathrm{~h}$ infection with $100 \mathrm{HU}$ NDV Ulster per $10^{7}$ cells (data not shown). Binding of the constructs to the IL-2 receptor was tested with IL-2 receptor-positive murine Eb lymphoma cells (28). The cells were first incubated with the recombinant proteins and then stained with mouse anti-His6-tag or mouse anti-E-tag followed by PE-labelled goat $\mathrm{F}\left(\mathrm{ab}^{\prime}\right)_{2}$ anti-mouse IgG and analyzed by flow cytometry. As to be expected, protein no. 766 , in contrast to protein no. 774 , bound to the Eb cells (Fig. 2, bottom panel).

The biological activity of the cytokine part of the fusion proteins was tested using IL-2-growth factor-dependent CTLL cells and GM-CSF growth factor-dependent TF-1 cells. The protein to be tested was serially diluted and tested for its capacity to facilitate the proliferation of the respective indicator cells as measured by ${ }^{3} \mathrm{H}$-thymidine uptake. As internal standards the serial dilutions of recombinant IL-2 and GM-CSF standards were used. Such calibration curves allowed to quantify the cytokine activity of the fusion proteins.

Table I summarizes the properties defined for both proteins. The fusion protein no. 766 contained $80299 \mathrm{U}$ per $\mu$ g protein and $16.12 \mathrm{ng}$ equivalent GM-CSF per $\mu \mathrm{g}$. The fusion protein no. 774 had no IL-2 activity and 2.37 equivalent ng GM-CSF per $\mu \mathrm{g}$ protein whereas protein no. 356 expressed only 51,852 units IL-2 activity per $\mu \mathrm{g}$ of protein (Table I).

Bystander anti-tumor activity induced in PBMC by GM-CSF is expressed at the cell surface of NDV-infected tumor vaccine. The functional activity of protein no. 774 (bsF-GMCSF) was evaluated in an in vitro tumor neutralisation assay (TNA) (27). This is basically a mixed lymphocyte tumor cell culture (MLTC) performed on top of an adherent tumor cell monolayer as bystander target. Co-incubation of PBMC for 5-7 days with the modified vaccine cells resulted in the generation of cytotoxic effector function and in the release of a variety of cytokines. We investigated the effects of the presence of the fusion protein bsF-GMCSF at various concentrations (from 163 to 2,611 ng eq. GM-CSF/well) (Fig. 3). This allowed to analyze the activity of this fusion protein on the PBMC: i) as soluble protein (white bars), ii) as protein bound to the tumor vaccine cells in the absence (gray bars) or presence (black bars) of a suboptimal $\mathrm{T}$ cell activation signal provided via bsHN-CD3 (27).

Fig. 3 shows the results obtained with two different PBMC donors. For the first donor, there was no difference in the antitumor capacity of PBMC activated via bsF-GMCSF bound to the tumor vaccine in presence or absence of the bsHN-CD3 protein. In presence of NDV-infected vaccine cells, the protein bsF-GMCSF induced, at the highest concentration, a $34 \%$ increase in tumor growth inhibition (TGI). In absence of the vaccine cells, the same quantity of bsF-GMCSF protein on PBMC led to a 5\% decrease of TGI.

For the second donor, the vaccine-bound bsF-GMCSF protein appeared to act as co-stimulatory molecule. Only in combination with a suboptimal concentration of bsHN-CD3 (700 pg/well), the measured anti-tumor activity was greatly increased (black bars). The presence of the tumor vaccine always increased the capacity of bsF-GMCSF to stimulate anti-tumor activity in PBMCs.

Activation of monocytes through addition of the recombinant protein bsF-GMCSF. Having shown that protein no. 774 is functionally active, it was of interest to find out which type of cell of the total PBMC responds to the addition of GM-CSF. Since monocytes are known to express the GM-CSF receptor, we performed a TNA test with purified monocytes and compared their antitumor effect to that obtained with whole PBMC or to that of PBMC depleted of monocytes. CD14 $4^{+}$monocytes, which account for 6 to $10 \%$ of the total PBMC, were magnetically purified as described in Materials and methods. The remaining PBMC population was entirely depleted of CD14positive cells. It was also verified that the $\mathrm{CD} 14^{+}$monocytes 
(2)

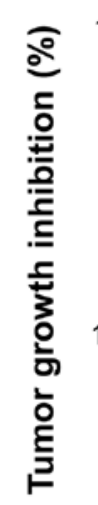

(3)

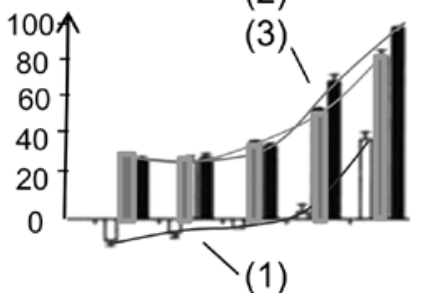

(1)

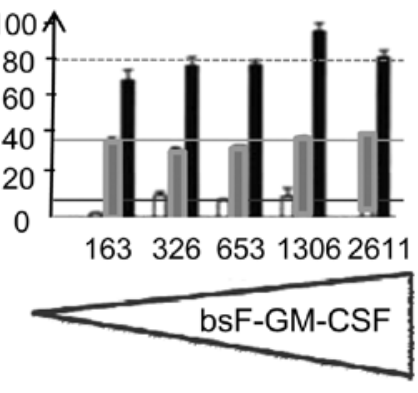

Donor 1

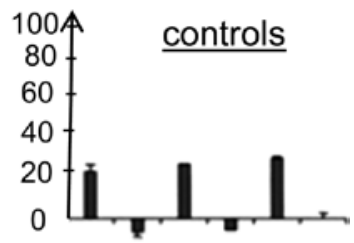

Donor 2

(3)

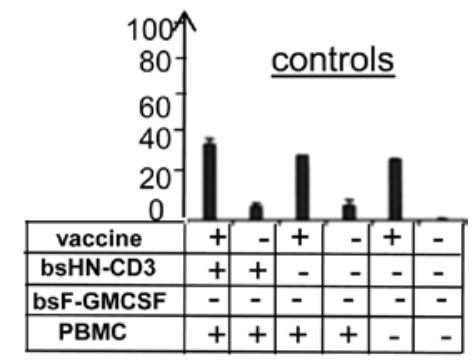

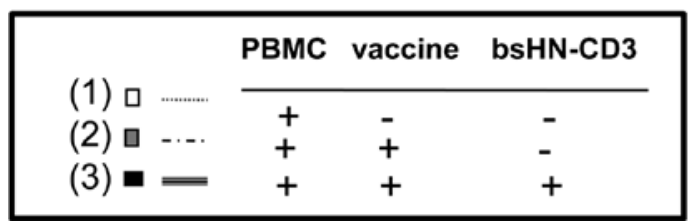

Figure 3. Induction of anti-tumor effects in PBMC by the fusion protein bsF-GMCSF (774) bound to the tumor vaccine in combination with suboptimal signal 1. Live MCF-7 cells (1.4x104/well) were seeded in a 48 -well plate and left for $4 \mathrm{~h}$ at $37^{\circ} \mathrm{C}$. Then, $3 \times 10^{4}$ human PBMC and $6 \times 10^{3} \mathrm{MCF}-7$ cells which were $200 \mathrm{~Gy}$ irradiated and modified with $100 \mathrm{HU}$ NDV Ulster/10 7 cells, were added per well. The activation of PBMC was carried out with a bsHN-CD3 concentration inducing sub-optimal signal 1 (700 pg/well) and various concentrations of bsF-GMCSF. Three conditions were tested on PBMC: i) addition of bsF-GMCSF on PBMC (white bars), ii) addition of bsF-GMCSF bound to the tumor vaccine (gray bars), and iii) addition of bsF-GMCSF bound to the tumor vaccine in the presence of suboptimal bs HN-CD3 (black bars). Intact MCF-7 cells served as control monolayers, which were incubated only with the vaccines, effector cells, or a combination of both. Other controls show the effect of the bsHN-CD3 fusion protein in the soluble form or as bound to the tumor vaccine (see controls on the right). After six days of incubation, the non-adherent cells were removed by suction, the wells filled with fresh medium and the MCF-7 monolayer were incubated in the dark for 45 min with $40 \mu 1$ MTS solution. The reaction was then stopped with $40 \mu 110 \%$ SDS solution and the result determined by measurement of optical density at $490 \mathrm{~nm}$. All values were determined as duplicates and are expressed as the mean \pm standard deviation. The symbols (+) and (-) indicate that the respective component in the test condition was respectively added or not. The results obtained with 2 different donors are shown as typical examples: donor 1 as expressing signal 1 and donor 2 as requiring signal 1.

expressed CD116, the GM-CSF receptor $\alpha$ chain, although at very low levels (Fig. 4Ac and d). Activation of the monocytes towards dendritic cells was tested by growth in the presence of recombinant interleukin-4 (IL-4, $10 \mathrm{ng} / \mathrm{ml}$ ) and 37 or $17 \mathrm{ng} / \mathrm{ml}$ GM-CSF (in the form of bsF-GMCSF). After three days of cell culture, monocytes, which were in contact with IL-4 and bsF-GM-CSF, showed massive proliferation and cluster formation which was not the case in control cultures (Fig. 4B).

Important role of monocytes in the bystander anti-tumor activity induced in PBMC by vaccine-attached GM-CSF. Highly purified monocytes ( $>99 \%$ CD14 positivity) were next tested in the TNA. The amount of bsF-GMCSF per well corresponds to $2.6 \mathrm{ng}$ GM-CSF. The monocytes were incubated either with soluble bsF-GM-CSF or with bsF-GMCSF bound to the tumor vaccine in the absence or presence of a suboptimal concentration of bsHN-CD3 (700 pg/well). For comparison, total PBMC and monocyte-depleted PBMC were evaluated in parallel. The result is shown in Fig. 5. Total PBMC (black bars) show a TGI of $30 \%$. This was increased to $42.5 \%$ through activation via bsF-GMCSF and to $83 \%$ after further addition of the tumor vaccine. The increase observed with bsF-GMCSF was independent of the CD3 signal, since the addition of bsHN-CD3 did not lead to a further increase of the TGI. Control cultures containing effector cells and vaccine without the recombinant protein showed a TGI of $28 \%$. The results obtained with purified monocytes (gray bars) show a rather similar picture, although the intensity is somewhat lower to the one obtained with total PBMC. The monocyte-depleted PBMC (white bars) showed for all three conditions of culture no difference to the controls. Thus, $\mathrm{CD} 14^{+}$monocytes appear as the main effector cells responsible for the effect obtained with the vaccine-attached fusion protein bsF-GMCSF.

Increased anti-tumor bystander effects upon co-stimulation of $P B M C$ with vaccine-attached $G M-C S F$ and $I L-2$. To analyze the antitumor effect of protein no. 766 (containing GM-CSF and IL-2) in comparison to protein no. 774 (containing only IL-2), the two proteins were titrated and tested in a TNA with PBMC. The results (Fig. 6) show first that the coupling of bsHN-CD3 (sub-optimal signal 1) led to $20 \%$ TGI (see controls). If 806 units IL-2 were added per well (either in the form of tsHN-IL2-GMCSF or bsHN-IL2), the TGI value 
A

(a)

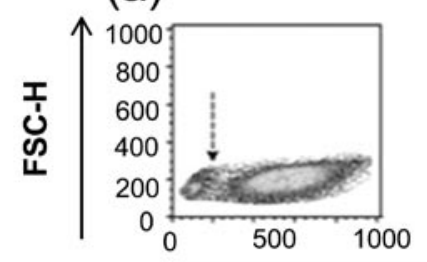

(b)

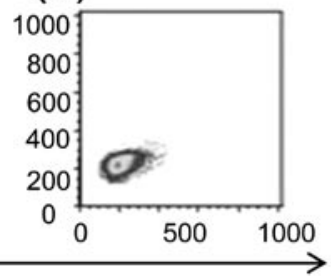

SSC-H

(c)
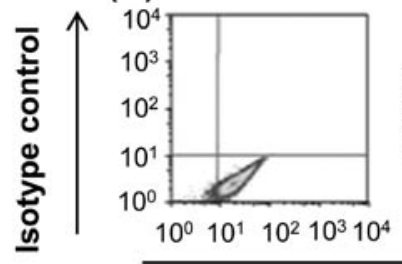

(d)

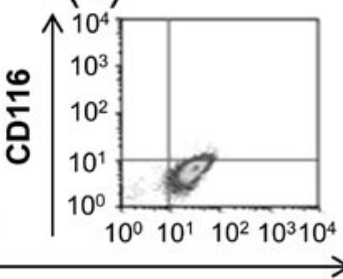

CD14

B (a)

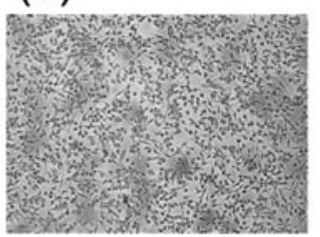

(c)

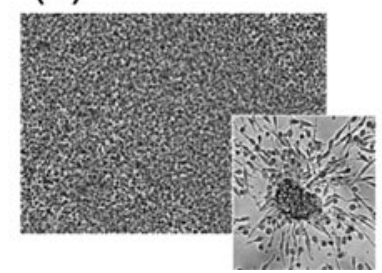

(b)

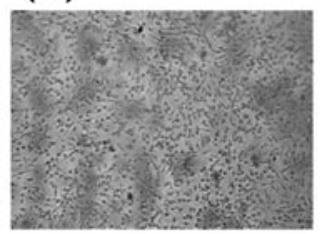

(d)

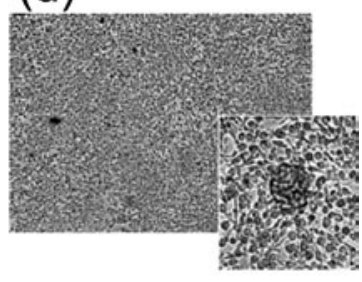

Figure 4. Biological activity of the protein bsF-GMCSF. (A) Purity of human monocytes ( $a$ and $b$ ) and expression of the GM-CSF receptor (CD116) at the cell surface of the monocytes (c and d). (a and b) $\mathrm{CD}_{14}{ }^{+}$monocytes from a healthy donor were purified from PBMC using magnetic beads as described in Materials and methods. To check their purity, $5 \times 10^{5}$ total PBMC, monocytedepleted PBMC or monocytes were blocked with Endobulin, then stained with FITC-conjugated anti-CD14 antibodies and analyzed by flow cytometry (data not shown). The depicted FSC/SSC scatter plot (a and b) confirms the purity of the monocytes. The population of monocytes is highlighted with an arrow. (c and d) Monocytes $\left(5 \times 10^{5}\right)$ were blocked with Endobulin and then stained with anti-CD14-FITC and biotinylated anti-CD116 antibody. The latter was detected with PE-conjugated streptavidin. The analysis was performed on FACS. Samples, which were stained only with anti-CD14-FITC and PE-conjugated streptavidin (unstained) served as controls. Dead cells were excluded from the measurement by PI staining. (B) Proliferation activity of the fusion protein no. 774 on purified human monocytes. Purified CD14 ${ }^{+}$monocytes $\left(2 \times 10^{6}\right)$ were incubated at $37^{\circ} \mathrm{C}$ with medium (a), $10 \mathrm{ng} / \mathrm{ml}$ recombinan IL-4 (b) or with $10 \mathrm{ng} / \mathrm{ml}$ recombinant IL-4 in the presence of 34 (c) or 17 (d) $\mathrm{ng} / \mathrm{ml}$ of the protein bsF-GMCSF (no. 774). After three days, microscopic images were recorded with 100 -fold magnification. Cluster patterns are shown in small pictures (with 230-fold magnification).

was increased to $\sim 90 \%$. Thus, the two proteins at this high concentration showed no difference. However, if the amounts were reduced, the TGI was significantly higher for the protein tsHN-IL2-GMCSF (no. 766) (p<0.04 at $101 \mathrm{U} /$ well, p<0.005 at $50 \mathrm{U} /$ well). Since the two proteins differ only in GM-CSF, the enhanced activation of the PBMC seen with the protein no. 766 can only be attributed to a GM-CSF effect. When both constructs were used at high concentrations without suboptimal bsHN-CD3 or even without bsHN-CD3 and without tumor vaccine (left two groups of Fig. 6A), no significant TGI was observed. This means that at least the IL-2 cytokine functions in this assay system only in conjunction with anti-CD3 mediated signal 1 and thus as a costimulatory signal.

Collectively, the results presented in this study show that IL-2 and GM-CSF can be targeted and attached to the ATV-NDV tumor vaccine easily by the described new immunocytokines. Vaccine modified in this way shows in functional in vitro assays increased anti-tumor activity due to an effect of GM-CSF on monocytes and to a T cell costimulatory effect of IL-2.

\section{Discussion}

In the present study we show that IL-2 and GM-CSF, when expressed as fusion proteins at the surface of a virus-modified tumor vaccine, are capable of triggering strong anti-tumor activity in vitro under conditions of a suboptimal anti-CD3 mediated $\mathrm{T}$ cell activation signal. The new NDV binding fusion proteins described contribute to the optimization of the human tumor vaccine ATV-NDV.

We previously established a therapeutically active autologous virus-modified tumor vaccine (ATV-NDV) for post-operative anti-tumor vaccination of cancer patients (2). It is based on preclinical studies demonstrating anti-metastatic activity in animal tumor models (29). The paramyxovirus NDV that is used for the production of this vaccine, shows tumor selectivity in its replication behavior (24). Through viral replication, new viral $\mathrm{HN}$ and $\mathrm{F}$ molecules become expressed at the surface of the tumor vaccine cells (9). These can serve as universal anchor molecules for the coupling of anti-HN or anti-F singlechain antibodies (scFv) (9). When such NDV-specific scFv reagents were fused with anti-CD3 $\mathrm{scFv}$ or with cytokines, we obtained either bispecific antibodies (e.g. bsHN-CD3) (25) or immunocytokines (e.g. bsHN-IL2) (26). The results obtained from in vitro experiments revealed that this type of tumor vaccine can activate PBMC to strong anti-tumor activity. Such modified vaccine might become very useful for the treatment of virtually any type of human cancer, provided that the patientderived cancer cells are susceptible to infection by NDV.

Recombinant antibody-cytokine fusion proteins (immunocytokines) can target tumors and achieve high cytokine concentrations in the tumor microenvironment (30). IL-2 immunocytokines targeted to tumor-associated antigens (TAAs) were shown in syngeneic mouse tumor models to be capable of eradicating established pulmonary and hepatic metastases of melanoma (31) and colorectal carcinoma (32). The immunocytokines showed biological activities of their cytokines since they were able to induce a dosedependent proliferation of either IL-2-dependent CTLL cells or GM-CSF-dependent TF1 cells. The IL-2 activity of our fusion proteins 766 and 356 ranged from $1.4 \times 10^{6}$ to $8.6 \times 10^{6} \mathrm{U} /$ ml. Similar activity was described by Neal et al (33) for a humanized anti-GD2 monoclonal antibody to which an IL-2 
A

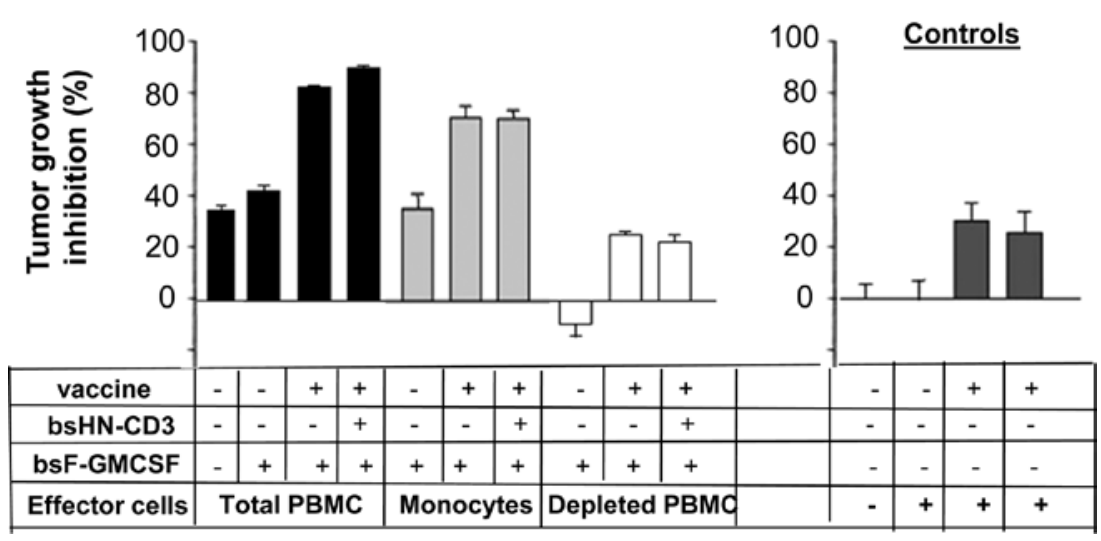

Figure 5. Importance of the monocytes fraction for the tumor growth inhibition induced by the GM-CSF cytokine activity on the surface of the tumor vaccine. Live MCF-7 cells were seeded on a 48-well plate (1.4x10 well) and incubated with 6x10 200 Gy $\gamma$-irradiated MCF-7 cells which were modified beforehand with $100 \mathrm{HU}$ NDV Ulster $/ 10^{7}$ cells as described in Materials and methods. As effector cells were added either total PBMC (black bars), purified $\mathrm{CD}_{4}{ }^{+}$monocytes (gray bars) or monocyte-depleted PBMC; white bars (A). The activation of effector cells was carried out in the presence of a sub-optimal dosis of a bsHN-CD3 (700 pg/well) and with $2.6 \mathrm{ng}$ of the construct bsF-GMCSF. Three conditions were tested: i) addition of bsF-GMCSF, ii) addition of tumor vaccine + bsF-GMCSF and iii) addition of tumor vaccine + bsF-GMCSF in the presence of suboptimal bsHN-CD3. Wells with intact monolayers of MCF-7 cells (to which only medium was added) served as control monolayers. Addition of each component separately [vaccines, effector or a combination of both (in the presence of bsHN-CD3 or not)] was also tested (B). After six days of incubation, the number of viable MCF-7 cells was evaluated as in Fig. 3 . All values were determined as triplicates, and are as the mean \pm standard deviation. (+) and (-) indicate that the respective component in the test condition was added or absent.

A

$\square$ bsHN-IL2 (\#356)

- tsHN-IL2-GMCSF (\#766)
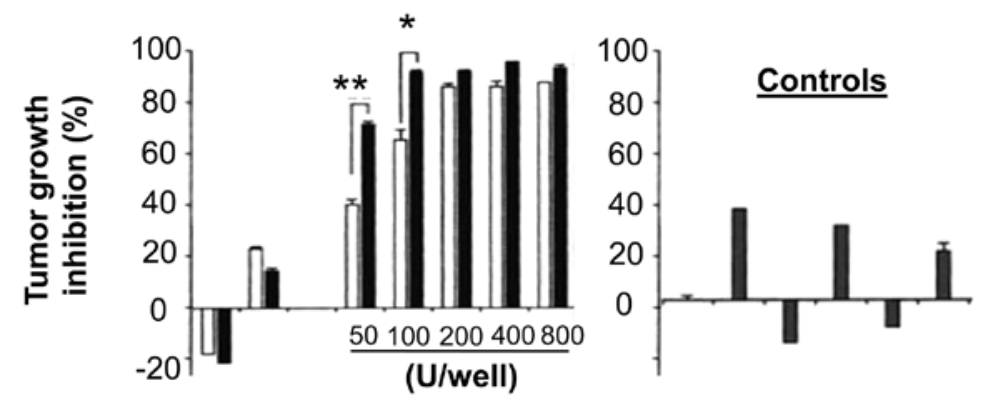

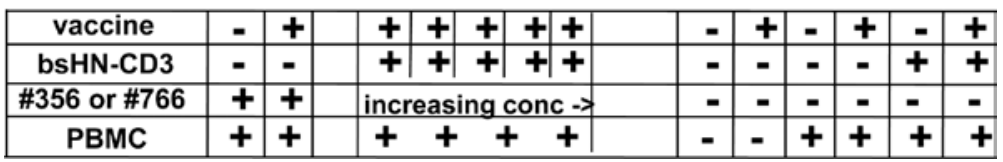

Figure 6. Better antitumor activities with bsHN-IL2-GMCSF than with bsHN-IL2 as bound to the tumor vaccine. Live MCF-7 cells (1.4x10 4 per well of 48 -well plate) were prepared and incubated with $6 \times 10^{3}{ }^{200}$ Gy-irradiated MCF-7 cells which were modified beforehand with 100 HU NDV Ulster/10 ${ }^{7}$ cells as described in Materials and methods. As effector cells were added either total PBMC (black bars), purified CD14+ monocytes (gray bars) or monocyte-depleted PBMC (white bars). The activation of effector cells was carried out on sub-optimal bsHN-CD3 (700 pg/well) and with bsHN-IL2-GMCSF (black bars) or bsHN-IL2 (white bar). Both stimulatory constructs were used as concentrations having the same IL-2 activities (A). Wells with intact monolayers MCF-7 cells (to which only medium was added) served as control monolayers. Addition of each components separately [vaccines, effector or a combination of both (in the presence of bsHN-CD3 or not)] was also tested (B). After six days of incubation, the number of viable MCF-7 cells was evaluated as in Fig. 2. All values were determined as triplicates, and are as mean \pm standard deviation. $(+)$ and (-) indicate that the respective component in the test condition was added or absent. ${ }^{*} \mathrm{p}<0.04,{ }^{* *} \mathrm{p}<0.005$.

gene was fused at the $\mathrm{C}$-termini of the two IgG1 heavy chains: hu14.18-IL2. This showed an IL-2 activity of $3.0 \times 10^{6} \mathrm{U} / \mathrm{ml}$. Normalized to the molecular weight, however, our constructs had stronger IL-2 activity $(6,800-80,100 \mathrm{U} / \mu \mathrm{g}$ total protein compared to $3,000 \mathrm{U} / \mu \mathrm{g}$ for the hu14.18-IL2).

The GM-CSF activity of the construct tsHN-IL2-GMCSF $(1,700 \mathrm{ng} / \mathrm{ml})$ corresponded well with the reference value obtained during the production of recombinant human GM-CSF in $\mathrm{CHO}$ cells with a yield of nearly $1,800 \mathrm{ng} / \mathrm{ml}$. The observation that the protein bsF-GMCSF showed a low concentration $(170 \mathrm{ng} / \mathrm{ml})$, should be attributed to the low level of expression of the F-specific constructs in the $\mathrm{CHO}$ expression system.

Overall, these data suggest that the individual components of the fusion proteins were correctly folded during their synthesis, without affecting each other negatively. In this respect, the linker connecting the individual components, 
consisting of four glycines and a serine (Gly4Serl), allowed for maximal flexibility. Steric effects between the scFv and the cytokine(s) were thus avoided $(34,35)$.

Our study aimed at improving the tumor vaccine ATV-NDV. Addition of fusion proteins to the tumor vaccine is expected to augment its anti-tumor activity. In addition to the previously described constructs that contain the essentials for T-cell activation: bsHN-CD3 and bsHN-CD28 $(25,27)$, we described in this work for the first time NDV binding fusion proteins which express the cytokines IL-2 and GM-CSF. Integration of these immunomodulatory cytokines into the tumor vaccine enhanced its capacity for activation of PBMC to exert bystander anti-tumor activity.

GM-CSF promotes the recruitment, differentiation and activation of granulocytes, macrophages/monocytes and eosinophil progenitor cells (19). A key feature of GM-CSF is the stimulation of immature dendritic cells that are considered the most effective antigen-presenting cells (36-38). These cells in the periphery internalize extracellular antigens (e.g. peptides, apoptotic and necrotic cell material, viruses and bacteria) and present the processed peptides in the context of MHC class II molecules and through so-called cross-presentation also in the context of MHC class-I molecules. Such professional antigen presenting cells then migrate into secondary lymphoid organs (draining lymph node) and induce specific T- and B-cell-based immune responses $(39,40)$. Thus, GM-CSF can affect not only innate immunity, but also indirectly adaptive (cellular and humoral) immunity.

Dranoff et al $(24,41)$ showed in a murine melanoma system, that GM-CSF-transduced melanoma cells were by far the best vaccine for the generation of protective immunity. This was based on strong recruitment of dendritic cells, macrophages and granulocytes to the vaccination site $(24,42)$. Promising results were also obtained in a clinical phase I study for the treatment of patients with metastatic melanoma. Immunohistochemical analysis of metastases of patients showed a stronger infiltration by $\mathrm{T}$ and $\mathrm{B}$ cells as well as an increased tumor shrinkage (43).

Our results of the TNA experiments with the 2 GM-CSF-containing immunocytokines, bsF-GMCSF and tsHN-GMCSF-IL2, can be summarized as follows: i) Soluble GM-CSF (in the form of bsF-GMCSF cells without the vaccine) has at high concentrations $(>6.5 \mathrm{ng} / \mathrm{ml})$ an activating effect on PBMC, which leads to tumor growth inhibition of a human MCF-7 tumor cell monolayer. ii) Antitumor activity mediated through GM-CSF could be further increased if the cytokine was linked to the tumor vaccine. Vaccine bound GM-CSF can be expected to bind to GM-CSF receptor-positive cells, monocytes, granulocytes, immature dendritic cells, and to reinforce interactions of such cells with the tumor vaccine. This could result in activation of these PBMC subpopulations. Our data are consistent with the results of Yei et al (44), who demonstrated in a murine melanoma model that vaccine cells that expressed GM-CSF in a membrane-bound form, were superior to GM-CSF secreting vaccine cells. iii) When stimulated with GM-CSF expressing vaccine cells, purified $\mathrm{CD} 14^{+}$ monocytes exerted in the TNA strong bystander anti-tumor activity. Total PBMC were somewhat stronger in such activity suggesting additional mechanisms. Since neither soluble, nor membrane-bound GM-CSF activated the monocyte-depleted
PBMC, other GM-CSF-sensitive cell types (such as granulocytes or dendritic cells) would merit to be tested for their eventual contribution to the antitumor effect observed. iv) The co-administration of IL-2 and GM-CSF (in the form of tsHNIL2-GMCSF) led to a greater antitumor effect in the TNA than each of the two cytokines alone. With respect to IL-2, the experimental conditions show the importance of the presence of a suboptimal $\mathrm{T}$ cell activation signal 1 via bsHN-CD3 for its antitumor effect. From this observation and further studies, we conclude that the effect of vaccine-bound IL-2 is exerted through CD25 (IL-2 receptor $\alpha$ chain) mediating a T cell costimulatory signal (45). Thus, the vaccine-bound cytokines IL-2 and GM-CSF affect different cell fractions of PBMC. This conclusion is corroborated by a study with GM-CSF/ IL-2-containing anti-EpCAM antibodies which showed a strong PBMC-mediated lysis of EpCAM-expressing tumor cells via two separate mechanisms (46). Our results are also in agreement with another study in a murine melanoma model in which the administration of vaccine cells which secreted IL-2/GM-CSF-fusion proteins, induced stronger anti-tumor immune responses than the separate application of IL-2- or GM-CSF-producing vaccine cells. Even a combination of equimolar concentrations of IL-2 and GM-CSF was not as effective as the monomer fusion protein (50). The favorable (immuno-) pharmacological properties of fusion proteins containing IL-2 and GM-CSF cytokines could be due to an increased resistance to proteolytic degradation in vivo. They could also be due to stronger activating effects of fusion proteins expressing both cytokines (IL-2 and GM-CSF) together.

The mechanisms through which the lysis of a tumor cell monolayer by monocytes occur, could not be defined yet by the presented data. However, since it is known that NDV induces in monocytes the expression of TRAIL (TNF-related apoptosis-inducing ligand) (6) and stimulates the production of anti-tumor agents such as TNF- $\alpha$ and NO (48), it can be speculated that these molecules are responsible for observed destruction of tumor cell monolayers. Monocytes could also destroy tumor cells through Fas-mediated apoptosis or through phagocytosis.

In this study, it was demonstrated that an extension of the tumor vaccine ATV-NDV by surface bound new fusion proteins that contain the cytokines IL-2 and GM-CSF results in increased in vitro antitumor activity by co-cultured PBMC. Due to the fact that IL-2 and GM-CSF activate different PBMC subsets, synergistic effects between cells from the innate and the adaptive immune system are likely to have been achieved. Recent data have demonstrated that IL-2 signals during priming endow $\mathrm{CD} 8^{+} \mathrm{T}$ cells with a memory program and secondary expansion capacity (49). The central importance of IL-2 in initiating adaptive immune responses is also well illustrated by the immunosuppressive effect on transplant rejection by rapamycin, a drug which inhibits signalling through the IL-2 receptor (50). It makes sense that immune effector cells are activated more efficiently if the various stimuli of a vaccine are presented together and not separately, since in this way effector cells can better integrate the signals. The most effective vaccine in this study is ATV-NDV modified with bsHN-CD3, IL-2 and GM-CSF. Results of another study showed that a combination of three costimuli (B7-1, ICAM-1 and LFA-3) in the presence of signal 1 resulted in stronger 
T-cell activation than the use of only one or two of the co-stimulatory signal(s) $(51,52)$.

Overall, the results of this study suggest that an extension of the basic vaccine ATV-NDV by linking CD3 signals with IL-2 and GM-CSF-containing immunocytokines is promising for the generation of more potent effects. In comparison to genetically modified tumor vaccines, the here presented modular approach of attachment of bispecific monoclonal antibodies (mAbs) and immunocytokines has the advantage of easy qualitative and quantitative adjustment of costimuli according to the requirements in the patient.

\section{Acknowledgements}

This study was part of the $\mathrm{PhD}$ thesis of Maximilian Aigner under co-supervision by Professor Volker Schirrmacher and Dr Philippe Fournier. We are particularly grateful to Annette Arnold for technical assistance, especially for help in the production and purification of the recombinant proteins. The writing of this manuscript would not have been possible without the kind financial support of Dr Kleist Stiftung (Berlin, Germany) and Christian-Berndt Stiftung (Rostock, Germany). We gratefully acknowledge also support by the IOZK (www.iozk.de) (Cologne, Germany).

\section{References}

1. Liebrich W, Schlag P, Manasterski M, Lehner B, Stöhr M, Möller P and Schirrmacher V: In vitro and clinical characterisation of a Newcastle disease virus-modified autologous tumour cell vaccine for treatment of colorectal cancer patients. Eur J Cancer 27: 703-710, 1991.

2. Schirrmacher V: Clinical trials of antitumor vaccination with an autologous tumor cell vaccine modified by virus infection: improvement of patient survival based on improved antitumor immune memory. Cancer Immunol Immunother 54: 587-598, 2005.

3. Ockert D, Schirrmacher V, Beck N, Stoelben E, Ahlert T, Flechtenmacher J, Hagmüller E, Buchcik R, Nagel M and Saeger HD: Newcastle disease virus-infected intact autologous tumor cell vaccine for adjuvant active specific immunotherapy of resected colorectal carcinoma. Clin Cancer Res 2: 21-28, 1996.

4. Schulze T, Kemmner W, Weitz J, Wernecke KD, Schirrmacher V and Schlag PM: Efficiency of adjuvant active specific immunization with Newcastle disease virus modified tumor cells in colorectal cancer patients following resection of liver metastases: results of a prospective randomized trial. Cancer Immunol Immunother 58: 61-69, 2009.

5. Fournier P, Zeng J and Schirrmacher V: Two ways to induce innate immune responses in human PBMCs: paracrine stimulation of IFN-alpha responses by viral protein or dsRNA. Int J Oncol 23: 673-680, 2003.

6. Zeng J, Fournier P and Schirrmacher V: Induction of interferonalpha and tumor necrosis factor-related apoptosis-inducing ligand in human blood mononuclear cells by hemagglutininneuraminidase but not $\mathrm{F}$ protein of Newcastle disease virus. Virology 297: 19-30, 2002.

7. Ertel C, Millar NS, Emmerson PT, Schirrmacher V and von Hoegen P: Viral hemagglutinin augments peptide-specific cytotoxic $\mathrm{T}$ cell responses. Eur J Immunol 23: 2592-2596, 1993.

8. Termeer CC, Schirrmacher V, Brocker EB and Becker JC: Newcastle disease virus infection induces B7-1/B7-2-independent T-cell costimulatory activity in human melanoma cells. Cancer Gene Ther 7: 316-323, 2000.

9. Fournier P, Aigner $M$ and Schirrmacher V: Optimization studies for the coupling of bispecific antibodies to viral anchor molecules of a tumor vaccine. Int J Oncol 37: 1203-1217, 2010.

10. Fiola C, Peeters B, Fournier P, Arnold A, Bucur M and Schirrmacher V: Tumor selective replication of Newcastle disease virus: association with defects of tumor cells in antiviral defence. Int J Cancer 119: 328-338, 2006.
11. Sule NS, Nerurkar RP and Kamath S: Interleukin-2 as a therapeutic agent. J Assoc Physicians India 49: 897-900, 2001.

12. Arai KI, Lee F, Miyajima A, Miyatake S, Arai N and Yokota T: Cytokines: coordinators of immune and inflammatory responses. Annu Rev Biochem 59: 783-836, 1990.

13. Rosenberg SA, Yang JC, White DE and Steinberg SM: Durability of complete responses in patients with metastatic cancer treated with high-dose interleukin2: identification of the antigens mediating response. Ann Surg 228: 307-319, 1998.

14. Yang JC, Haworth L, Sherry RM, Hwu P, Schwartzentruber DJ, Topalian SL, Steinberg SM, Chen HX and Rosenberg SA: Randomized study of high-dose and low-dose interleukin-2 in patients with metastatic renal cancer. J Clin Oncol 21: 3127-3132, 2003.

15. Lotze MT, Chang AE, Seipp CA, Simpson C, Vetto JT and Rosenberg SA: High-dose recombinant interleukin 2 in the treatment of patients with disseminated cancer. Responses, treatment-related morbidity, and histologic findings. JAMA 256: 3117-3124, 1986.

16. Bruton JK and Koeller JM: Recombinant interleukin-2. Pharmacotherapy 14: 635-656, 1994.

17. Xu X, Clarke P, Szalai G, Shively JE, Williams LE, Shyr Y, Shi E and Primus FJ: Targeting and therapy of carcinoembryonic antigen-expressing tumors in transgenic mice with an antibodyinterleukin 2 fusion protein. Cancer Res 60: 4475-4484, 2000.

18. Ruef C and Coleman DL: Granulocyte-macrophage colonystimulating factor: pleiotropic cytokine with potential clinical usefulness. Rev Infect Dis 12: 41-62, 1990.

19. Pulendran B, Lingappa J, Kennedy MK, Smith J, Teepe M, Rudensky A, Maliszewski CR and Maraskovsky E: Developmental pathways of dendritic cells in vivo: distinct function, phenotype, and localization of dendritic cell subsets in FLT3 ligand-treated mice. J Immunol 159: 2222-2231, 1997.

20. Shen Z, Reznikoff G, Dranoff G and Rock KL: Cloned dendritic cells can present exogenous antigens on both MHC class I and class II molecules. J Immunol 158: 2723-2730, 1997.

21. Zou GM and Tam YK: Cytokines in the generation and maturation of dendritic cells: recent advances. Eur Cytokine Netw 13: 186-199, 2002.

22. Murtaza A, Kuchroo VK and Freeman GJ: Changes in the strength of co-stimulation through the B7/CD28 pathway alter functional $\mathrm{T}$ cell responses to altered peptide ligands. Int Immunol 11: 407-416, 1999.

23. Dranoff G, Jaffee E, Lazenby A, Golumbek P, Levitsky H, Brose K, Jackson V, Hamada H, Pardoll D and Mulligan RC: Vaccination with irradiated tumor cells engineered to secrete murine granulocyte-macrophage colony-stimulating factor stimulates potent, specific, and long-lasting anti-tumor immunity. Proc Natl Acad Sci USA 90: 3539-3543, 1993.

24. Schirrmacher V, Haas C, Bonifer R, Ahlert T, Gerhards R and Ertel C: Human tumor cell modification by virus infection: an efficient and safe way to produce cancer vaccine with pleiotropic immune stimulatory properties when using Newcastle disease virus. Gene Ther 6: 63-73, 1999.

25. Haas C, Lulei M, Fournier P, Arnold A and Schirrmacher V: T-cell triggering by CD3- and CD28-binding molecules linked to a human virus-modified tumor cell vaccine. Vaccine 23 : 2439-2453, 2005.

26. Bian H, Fournier P, Moormann R, Peeters B and Schirrmacher V: Selective gene transfer in vitro to tumor cells via recombinant Newcastle disease virus. Cancer Gene Ther 12: 295-303, 2005.

27. Haas C, Lulei M, Fournier P, Arnold A and Schirrmacher V: A tumor vaccine containing anti-CD3 and anti-CD28 bispecific antibodies triggers strong and durable antitumor activity in human lymphocytes. Int J Cancer 118: 658-667, 2006.

28. Diamantstein T and Osawa $\mathrm{H}$ : Studies on the interleukin-2 receptor, its generation and dynamics using monoclonal anti-interleukin-2 receptor antibodies. Mol Immunol 21: 1229-1236, 1984.

29. Heicappell R, Schirrmacher V, von Hoegen P, Ahlert T and Appelhans B: Prevention of metastatic spread by postoperative immunotherapy with virally modified autologous tumor cells. I. Parameters for optimal therapeutic effects. Int J Cancer 37: 569-577, 1986.

30. Lode HN, Xiang R, Becker JC, Gillies SD and Reisfeld RA: Immunocytokines: a promising approach to cancer immunotherapy. Pharmacol Ther 80: 277-292, 1998.

31. Becker JC, Pancook JD, Gillies SD, Mendelsohn J and Reisfeld RA: Eradication of human hepatic and pulmonary melanoma metastases in SCID mice by antibody-interleukin 2 fusion proteins. Proc Natl Acad Sci USA 93: 2702-2707, 1996. 
32. Xiang R, Lode HN, Dolman CS, Dreier T, Varki NM, Qian X, Lo KM, Lan Y, Super M, Gillies SD and Reisfeld RA: Elimination of established murine colon carcinoma metastases by antibodyinterleukin 2 fusion protein therapy. Cancer Res 57: 4948-4955, 1997.

33. Neal ZC, Yang JC, Rakhmilevich AL, Buhtoiarov IN, Lum HE, Imboden M, Hank JA, Lode HN, Reisfeld RA, Gillies SD and Sondel PM: Enhanced activity of hu14.18-IL2 immunocytokine against murine NXS2 neuroblastoma when combined with interleukin 2 therapy. Clin Cancer Res 10: 4839-4847, 2004.

34. Kufer P, Mack M, Gruber R, Lutterbuse R, Zettl F and Riethmuller G: Construction and biological activity of a recombinant bispecific single-chain antibody designed for therapy of minimal residual colorectal cancer. Cancer Immunol Immunother 45: 193-197, 1997.

35. Kufer P, Lutterbuse R and Baeuerle PA: A revival of bispecific antibodies. Trends Biotechnol 22: 238-244, 2004.

36. Hart DN: Dendritic cells: unique leukocyte populations which control the primary immune response. Blood 90: 3245-3287, 1997.

37. Wright-Browne V, McClain KL, Talpaz M, Ordonez $\mathrm{N}$ and Estrov Z: Physiology and pathophysiology of dendritic cells. Hum Pathol 28: 563-579, 1997.

38. Steinman RM: The dendritic cell system and its role in immunogenicity. Annu Rev Immunol 9: 271-296, 1991

39. Guermonprez P, Valladeau J, Zitvogel L, Thery C and Amigorena S: Antigen presentation and T cell stimulation by dendritic cells. Annu Rev Immunol 20: 621-667, 2002.

40. Banchereau J, Briere F, Caux C, Davoust J, Lebecque S, Liu YJ, Pulendran B and Palucka K: Immunobiology of dendritic cells. Annu Rev Immunol 18: 767811, 2000.

41. Dranoff G: GM-CSF-secreting melanoma vaccines. Oncogene 22: 3188-3192, 2003

42. Mach N, Gillessen S, Wilson SB, Sheehan C, Mihm M and Dranoff G: Differences in dendritic cells stimulated in vivo by tumors engineered to secrete granulocyte-macrophage colonystimulating factor or Flt3-ligand. Cancer Res 60: 3239-3246, 2000 .

43. Soiffer R, Lynch T, Mihm M, Jung K, Rhuda C, Schmollinger JC, Hodi FS, Liebster L, Lam P, Mentzer S, Singer S, Tanabe KK, Cosimi AB, Duda R, Sober A, Bhan A, Daley J, Neuberg D, Parry G, Rokovich J, Richards L, Drayer J, Berns A, Clift S, Cohen LK, Mulligan RC and Dranoff G: Vaccination with irradiated autologous melanoma cells engineered to secrete human granulocytemacrophage colony-stimulating factor generates potent antitumor immunity in patients with metastatic melanoma. Proc Natl Acad Sci USA 95: 13141-13146, 1998.
44. Yei S, Bartholomew RM, Pezzoli P, Gutierrez A, Gouveia E, Bassett D, Soo Hoo W and Carlo DJ: Novel membrane-bound GM-CSF vaccines for the treatment of cancer: generation and evaluation of mbGM-CSF mouse B16F10 melanoma cell vaccine. Gene Ther 9: 1302-1311, 2002.

45. Aigner M, Janke M, Lulei M, Beckhove P, Fournier P and Schirrmacher V: An effective tumor vaccine optimized for costimulation via bispecific and trispecific fusion proteins. Int J Oncol 32: 777-789, 2008

46. Schanzer JM, Baeuerle PA, Dreier T and Kufer P: A human cytokine/single-chain antibody fusion protein for simultaneous delivery of GM-CSF and IL-2 to Ep-CAM overexpressing tumor cells. Cancer Immun 6: 4, 2006.

47. Stagg, J, Wu JH, Bouganim N and Galipeau J: Granulocytemacrophage colony-stimulating factor and interleukin-2 fusion cDNA for cancer gene immunotherapy. Cancer Res 64: 8795-8799, 2004.

48. Schirrmacher V, Bai L, Umansky V, Yu L, Xing Y and Qian Z: Newcastle disease virus activates macrophages for anti-tumor activity. Int J Oncol 16: 363-373, 2000.

49. Williams MA, Tyznik AJ and Bevan MJ: Interleukin-2 signals during priming are required for secondary expansion of $\mathrm{CD} 8^{+}$ memory T cells. Nature 441: 890-893, 2006.

50. Dumont FJ, Melino MR, Staruch MJ, Koprak SL, Fischer PA and Sigal NH: The immunosuppressive macrolides FK-506 and rapamycin act as reciprocal antagonists in murine $\mathrm{T}$ cells. $\mathrm{J}$ Immunol 144: 1418-1424, 1990.

51. Schlom J, Sabzevari H, Grosenbach DW and Hodge JW: A triad of costimulatory molecules synergize to amplify T-cell activation in both vector-based and vector-infected dendritic cell vaccines. Artif Cells Blood Substit Immobil Biotechnol 31: 193-228, 2003.

52. Zhu M, Terasawa H, Gulley J, Panicali D, Arlen P, Schlom J and Tsang KY: Enhanced activation of human T cells via avipox vector-mediated hyperexpression of a triad of costimulatory molecules in human dendritic cells. Cancer Res 61: 3725-3734, 2001. 